\title{
Longitudinal development of EAS muon component - comparison of data from the Muon Tracking Detector in KASCADE-Grande with model predictions
}

\author{
P. Łuczak ${ }^{11}$, J. Zabierowski ${ }^{11}$, W.D. Apel ${ }^{1}$, J.C. Arteaga-Velázquez ${ }^{2}$, K. Bekk ${ }^{1}$, \\ M. Bertaina ${ }^{3}$, J. Blümer ${ }^{1,4}$, H. Bozdog ${ }^{1}$, I.M. Brancus ${ }^{5}$, E. Cantoni ${ }^{3,6}$, A. Chiavassa ${ }^{3}$, \\ F. Cossavella ${ }^{4}$, K. Daumiller ${ }^{1}$, V. de Souza ${ }^{7}$, F. Di Pierro ${ }^{3}$, P. Doll ${ }^{1}$, R. Engel ${ }^{1}$, \\ D. Fuhrmann ${ }^{8}$, A. Gherghel-Lascu ${ }^{5}$, H.J. Gils ${ }^{1}$, R. Glasstetter ${ }^{8}$, C. Grupen ${ }^{9}$, \\ A. Haungs ${ }^{1}$, D. Heck ${ }^{1}$, J.R. Hörandel ${ }^{10}$, D. Huber ${ }^{4}$, T. Huege ${ }^{1}$, K.-H. Kampert ${ }^{8}$, \\ D. Kang ${ }^{4}$, H.O. Klages ${ }^{1}$, K. Link ${ }^{4}$, H.J. Mathes ${ }^{1}$, H.J. Mayer ${ }^{1}$, J. Milke ${ }^{1}$, B. Mitrica ${ }^{5}$, \\ C. Morello ${ }^{6}$, J. Oehlschläger ${ }^{1}$, S. Ostapchenko ${ }^{12}$, N. Palmieri' ${ }^{4}$, T. Pierog ${ }^{1}$, H. Rebel $^{1}$, \\ M. Roth ${ }^{1}$, H. Schieler ${ }^{1}$,S. Schoo*1, F.G. Schröder ${ }^{1}$, O. Sima ${ }^{13}$, G. Toma ${ }^{5}$, \\ G.C. Trinchero ${ }^{6}$, H. Ulrich ${ }^{1}$, A. Weindl ${ }^{1}$, J. Wochele ${ }^{1}$ - KASCADE-Grande \\ Collaboration \\ ${ }^{1}$ Institut für Kernphysik, KIT - Karlsruhe Institute of Technology, Germany \\ ${ }^{2}$ Universidad Michoacana, Inst. Física y Matemáticas, Morelia, Mexico \\ ${ }^{3}$ Dipartimento di Fisica, Università degli Studi di Torino, Italy \\ ${ }^{4}$ Institut für Experimentelle Kernphysik, KIT - Karlsruhe Institute of Technology, Germany \\ ${ }^{5}$ Horia Hulubei National Institute of Physics and Nuclear Engineering, Bucharest, Romania \\ ${ }^{6}$ Osservatorio Astrofisico di Torino, INAF Torino, Italy \\ ${ }^{7}$ Universidade São Paulo, Instituto de Física de São Carlos, Brasil \\ ${ }^{8}$ Fachbereich Physik, Universität Wuppertal, Germany \\ ${ }^{9}$ Department of Physics, Siegen University, Germany \\ ${ }^{10}$ Dept. of Astrophysics, Radboud University Nijmegen, The Netherlands \\ ${ }^{11}$ National Centre for Nuclear Research, Department of Astrophysics, Lódź, Poland \\ ${ }^{12}$ Frankfurt Institute for Advanced Studies (FIAS), Frankfurt am Main, Germany \\ ${ }^{13}$ Department of Physics, University of Bucharest, Bucharest, Romania \\ E-mail: pawel.luczak@ncbj.gov.pl
}

\begin{abstract}
The purpose of this analysis is to investigate the production heights of muons $\left(\mathrm{E}_{\mu}^{t h r}>0.8 \mathrm{GeV}\right)$ in extensive air showers (EAS) with primary energies above $10^{16} \mathrm{eV}$, registered with the Muon Tracking Detector of KASCADE-Grande. The registered showers are compared with the simulations of EAS obtained with four high energy hadronic interaction models. The ability of the models to describe the experimental data is discussed.
\end{abstract}

The 34th International Cosmic Ray Conference,

30 July- 6 August, 2015

The Hague, The Netherlands

\footnotetext{
* Speaker.
} 


\section{Introduction}

One of fields of interest of the ultra high energy (UHE) astrophysics is the investigation of UHE cosmic ray (CR) particles interacting with the nuclei of the Earths atmosphere and creating extensive air showers (EAS). This phenomenon is studied e.g. by means of ground based experiments of large detection area, like the KASCADE-Grande [1]. A complex nature of the UHE CR particles can be understood by investigation of as many components of the EAS cascade as possible. One of the most important is the measurement of the muon component. The EAS muons, being a result of decays of mesons, created in hadronic interactions, carry information about those interactions to the observation level. In turn, knowledge of UHE hadronic interactions is crucial for answering many questions formulated in CR research.

The large area Muon Tracking Detector $\left(128 \mathrm{~m}^{2}\right)$ [2], one of the components of the KASCADEGrande experiment, has been built with the aim to identify muons $\left(\mathrm{E}_{\mu}^{t h r}>0.8 \mathrm{GeV}\right)$ and their angular correlation with the extensive air shower. Combining information about the direction of EAS, obtained with the KASCADE-Grande particle detector array, and the directions of reconstructed muon tracks we have investigated the muon production heights (MPH) by means of the triangulation method giving us a direct insight into the longitudinal development of the muonic component of EAS for showers with the primary energy above $10^{16} \mathrm{eV}$. The results of the previous MPH analysis done with the MTD data in energy range below $10^{16} \mathrm{eV}$ can be found in Ref. [3].

In this work, the distributions of measured muon production heights are compared to the distributions obtained for simulated showers. The simulations were obtained with the CORSIKA [4] code for proton and iron as the primary particles and for four high-energy hadronic interaction models: QGSJet-II-02 [5], QGSJet-II-04 [6], EPOS 1.99 [7] and EPOS-LHC [8], in combination with FLUKA [9] as a low-energy hadronic interaction model. The ability of the interaction models to reproduce the experimental results is presented and discussed.

\section{Selection of the data}

The investigation being a subject of this work is based on over 45 million showers registered by the KASCADE-Grande experiment from November 2003 to June 2009. Selection criteria for the showers in KASCADE-Grande were a subject of extended investigation. After detailed studies of the performance of the Grande Array a set of conditions, superimposed on measured and reconstructed shower parameters, was developed to obtain highest reconstruction accuracy and to minimize systematic errors [1]. For this analysis only the showers with zenith angle less than $18^{\circ}$ with at least one muon track reconstructed in the MTD are taken into account, more than 13000, in total.

The analysis is done in two primary energy intervals: $\lg (\mathrm{E}[\mathrm{GeV}])>7.0$ and $\lg (\mathrm{E}[\mathrm{GeV}])>7.6$, see Table 1 . The energy was estimated with the formula described in detail in Ref. [10]).

The registered showers have been divided into light and heavy samples (called light and heavy data). This selection was done using the mean value of the Y distribution $\left(Y=\lg \left(N_{\mu}\right) / \lg \left(N_{c h}\right)\right)$ for simulated showers where the primary particle is helium or silicon nuclei. The light data showers are those with $Y<\left\langle Y_{H e}\right\rangle$, the heavy data showers are those with $Y>\left\langle Y_{S i}\right\rangle$. Light and heavy 
Table 1: Energy ranges.

\begin{tabular}{|c|c|c|}
\hline $\lg (E[\mathrm{GeV}])$ & $>7.0$ & $>7.6$ \\
\hline$\langle E\rangle\left[10^{16} \mathrm{eV}\right]$ & 1.8 & 7.3 \\
\hline
\end{tabular}

data showers are compared with simulations, where the primary cosmic ray particles are hydrogen (called light simulations) and iron (called heavy simulations).

\section{Results and discussion}

In the first energy interval the comparison of the MPH distributions obtained for the registered and simulated showers reveals that none of the investigated models describes the data well. It is especially seen with the light data sample (Figure 1).

Light simulated showers, in our case initiated only by proton primaries, should develop deeper in the atmosphere than light data showers, which are a mixture of showers with primaries from proton and helium. As shown in Figure 1 the distributions obtained with the QGSJet and EPOS models are shifted with respect to the data. This behavior suggests that simulated showers develop earlier in the atmosphere than registered showers.

One can notice that the range of the shift is different among models, it is stronger for the QGSJet than for the EPOS model. It is well seen in Figure 3, where parts of the distributions shown in Figure 1 are presented in the way to make this effect better visible. The shift in distributions between data and simulations is the smallest for the EPOS-LHC model. This is an indication of an improvement in describing the shower development by this model in respect to the others.

With the increasing primary energy the mass composition of CRs is changing, the mean mass of primary particle of the registered showers is becoming heavier [11]. This results in the change in the relation between the MPH distributions of the registered and simulated showers (see Figure 2). Now the data points for the light sample (where the content of proton primaries became negligibly small) indicate the earlier development of muon component than for pure proton showers. The heavy data points are now also situated closer to the simulation results for pure iron primaries (heavy simulations). This can be seen for all investigated models.

The differences between models in relative positions of experimental and simulated MPH distributions are now smaller than at lower primary energy but the statistical errors are much larger now. Therefore, the better quality of the EPOS-LHC model with respect to the QGSJet models in describing the shower development is not seen here so clearly as in the case of the lower primary energy range. The observed shift between the light distributions in data and simulation indicates that registered showers develop slightly earlier in the atmosphere than simulated showers. This can be seen for all models (compare Figure 1 and Figure 2).

In case of heavy showers one observes a slightly later development in the atmosphere of heavy data compared to heavy simulations, but the differences between data and simulations for different models, especially in the higher shower energy range, cannot be seen so well as in case of the light sample, due to statistical errors.

It has to be pointed out that the observed improvement of the relation between the distributions 


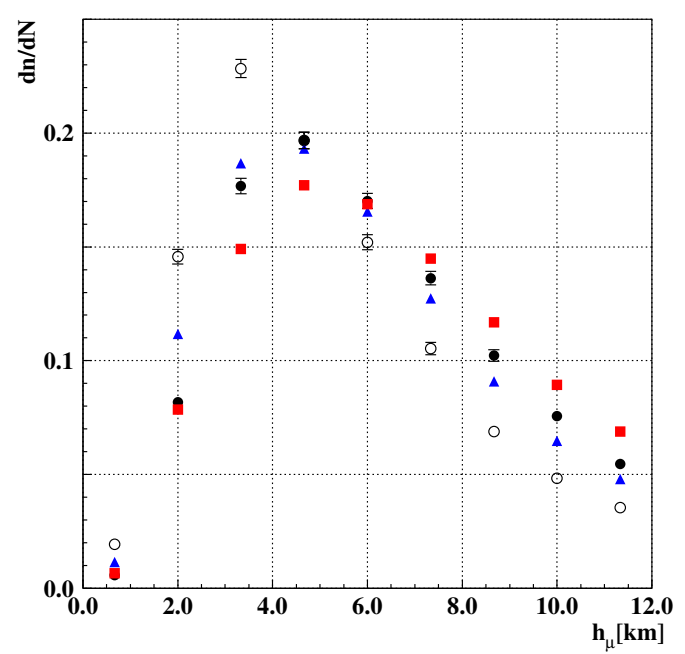

(a) QGSJet-II-02

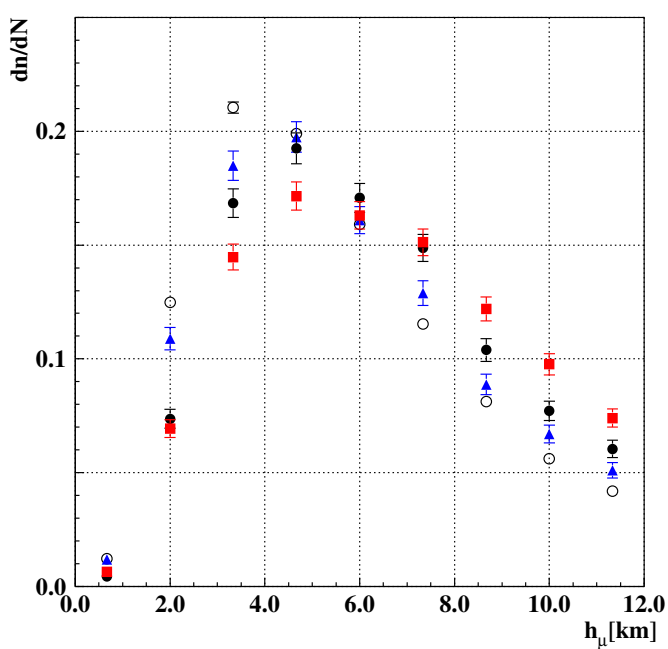

(c) EPOS 1.99

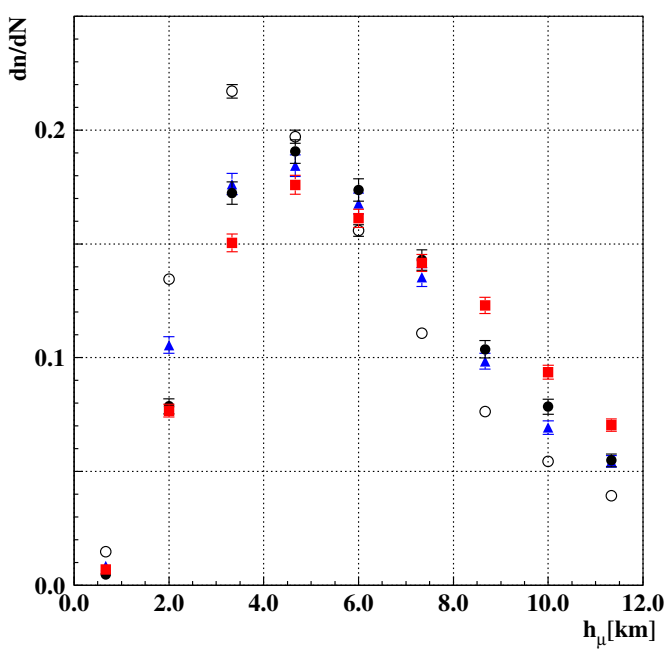

(b) QGSJet-II-04

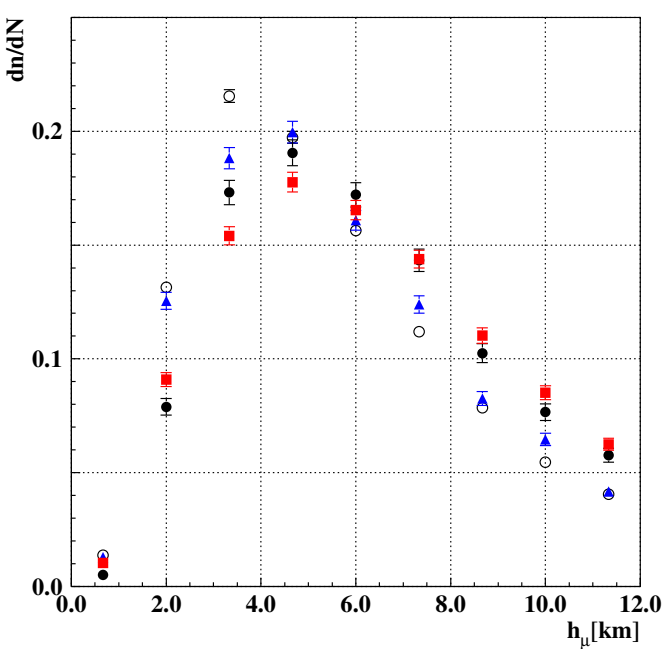

(d) EPOS-LHC

Figure 1: The MPH distributions from light and heavy data (open and solid circle, respectively) and light and heavy simulations (triangle and square, respectively) in range $\lg (\mathrm{E}[\mathrm{GeV}])>7.0$.

of simulated and registered showers is in large part rather caused not by the improved description of showers at higher energies but by the increase of the mean mass of the registered showers.

\section{Conclusions}

The investigation the of muon production height and comparison of its distributions obtained with the registered and simulated showers for four high-energy hadronic interaction models in two primary energy intervals was presented. The experimental data has been divided into light and 


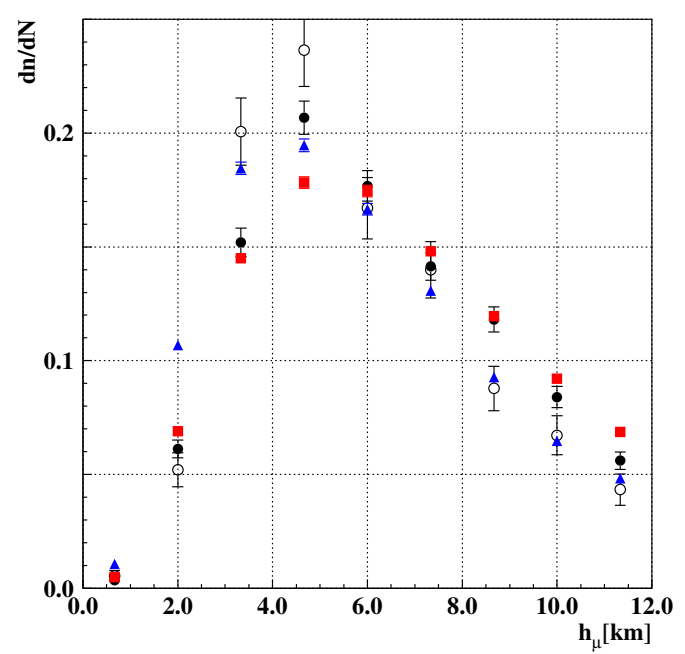

(a) QGSJet-II-02

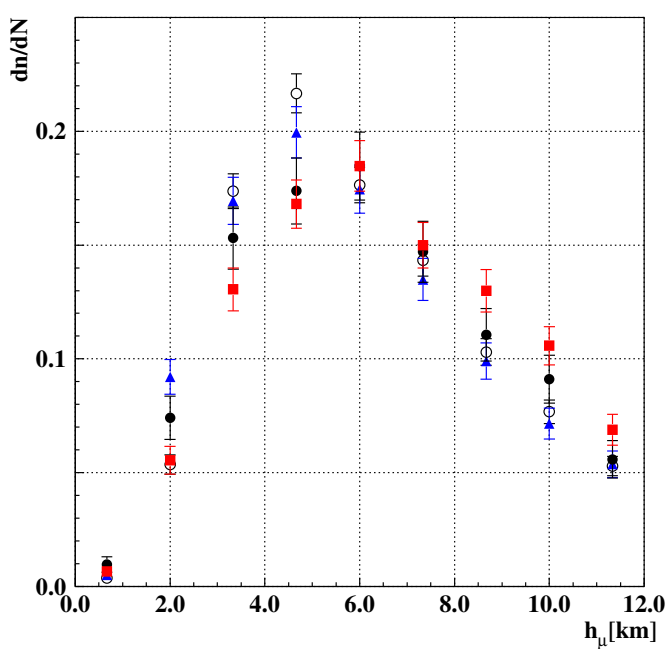

(c) EPOS 1.99

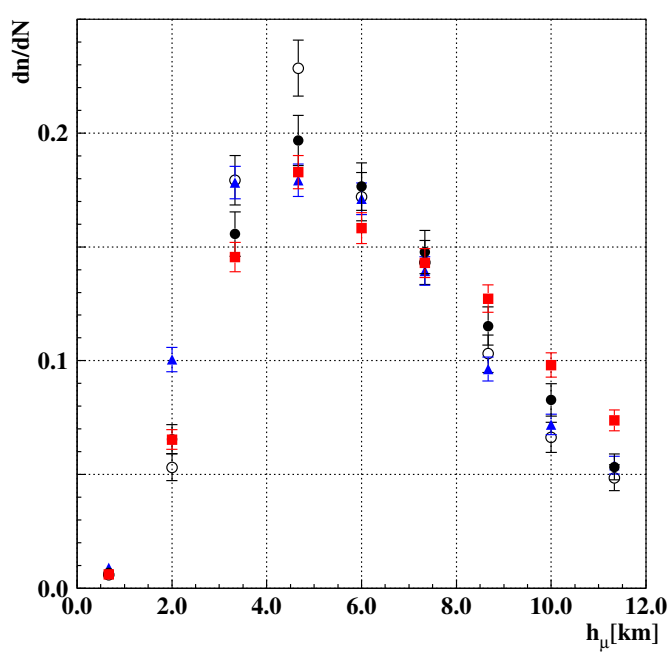

(b) QGSJet-II-04

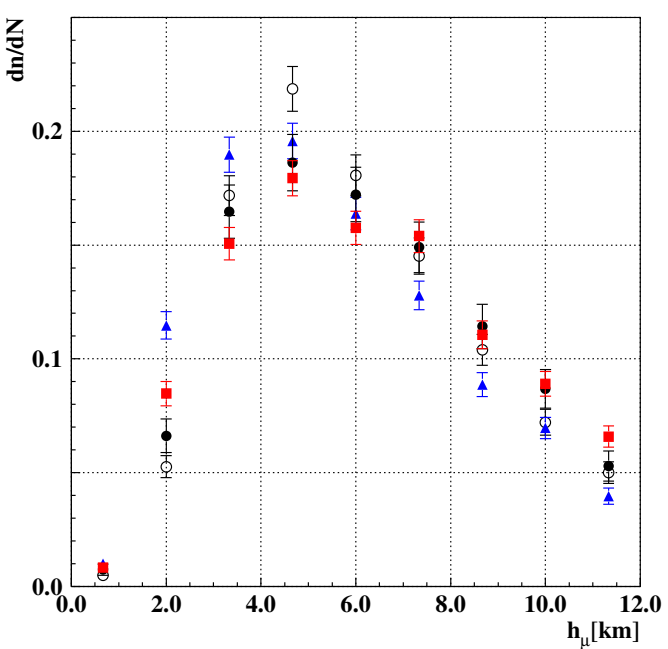

(d) EPOS-LHC

Figure 2: The MPH distributions from light and heavy data (open and solid circle, respectively) and light and heavy simulations (triangle and square, respectively) in range $\lg (\mathrm{E}[\mathrm{GeV}])>7.6$.

heavy samples.

All investigated interaction models do not describe the data correctly, what is especially well seen with the light data sample in the first, lower, primary energy interval. The behavior of the simulated distributions suggests that the light simulated showers develop earlier in the atmosphere than the measured ones. However, it has been shown that in comparison with the QGSJet models the EPOS-LHC model better describes the longitudinal development of the muon component in air showers. This is changing with the increase of the primary CR energy, as the mean mass compo- 


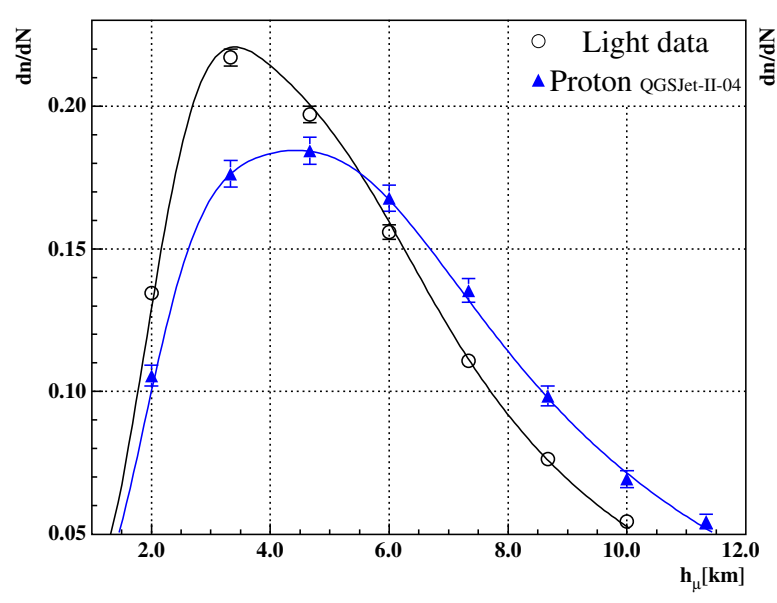

(a)

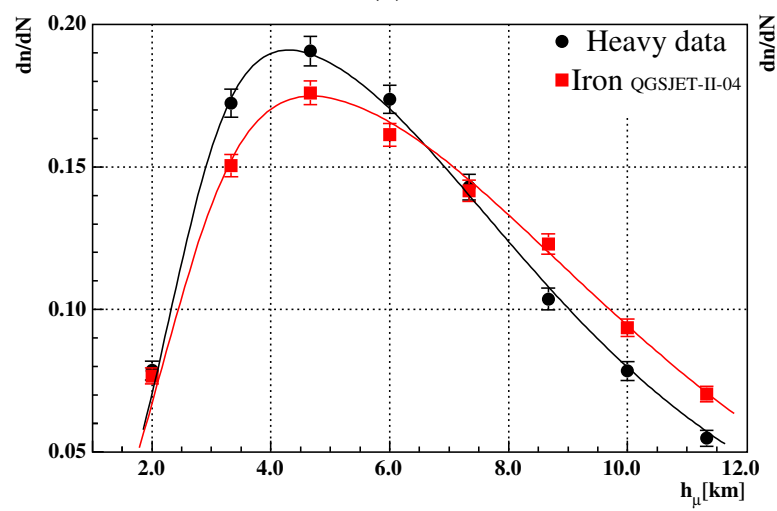

(c)

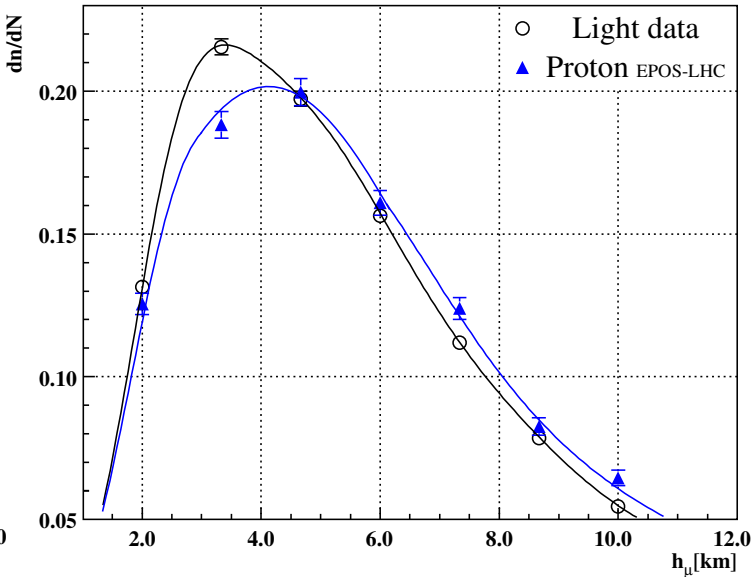

(b)

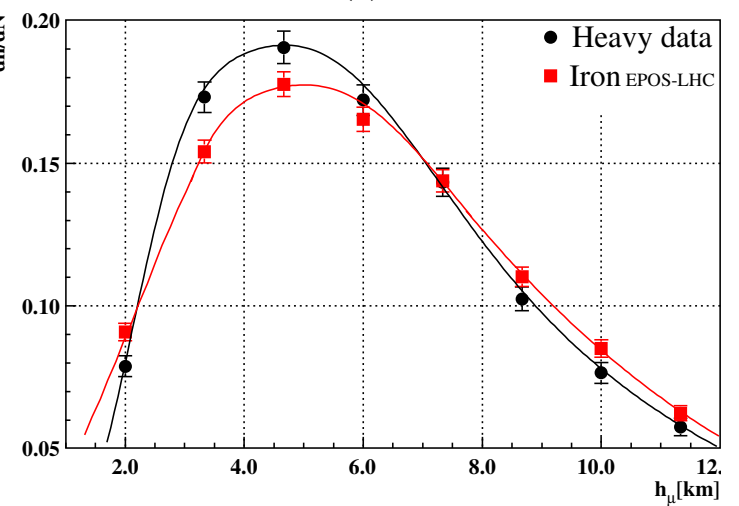

(d)

Figure 3: The MPH distributions in the primary energy range $\lg (\mathrm{E}[\mathrm{GeV}])>7.0$ : from light data (open circle) and H simulations (triangles) obtained with QGSJet-II-04 (a) and EPOS-LHC (b) and from heavy data (solid circles) and Fe simulations (squares) obtained with QGSJet-II-04 (c) and EPOS-LHC (d). Lines are drawn to guide the eye.

sition of the data showers is increasing, what is seen in the changed relations between the MPH distributions for experimental data and simulations.

The smallest differences between the light data and simulated showers can be observed for the EPOS-LHC model.

\section{References}

[1] W. A. et al. (KASCADE-Grande Collaboration), "The KASCADE-Grande experiment", Nucl. Instrum. Meth. A 620 (2010) 202-216.

[2] P. Doll, W. Bartl, C. Büttner, K. Daumiller, K. Kampert, H. Klages, D. Martello, R. Obenland, L. Pentchev, and J. Zabierowski, "Muon tracking detector for the air shower experiment KASCADE", Nucl. Instrum. Meth. A 488 (2002), no. 3517 - 535. 
[3] W. A. et al. (KASCADE-Grande Collaboration), "Muon production height studies with the air shower experiment KASCADE-Grande”, Astropart. Phys. 34 (2011), no. 6476 - 485.

[4] D. H. et al., “CORSIKA: a Monte Carlo code to simulate extensive air showers”, Tech. Rep. 6019, Forschungszentrum Karlsruhe(FZKA), 1998.

[5] S. Ostapchenko, "Nonlinear screening effects in high energy hadronic interactions", Phys.Rev.D 74 (2006) 014026.

[6] S. Ostapchenko, "Monte Carlo treatment of hadronic interactions in enhanced Pomeron scheme: QGSJET-II model”, Phys.Rev.D 83 (2011) 014018.

[7] T. Pierog and K. Werner, "EPOS Model and Ultra High Energy Cosmic Rays”, Nucl.Phys.Proc.Suppl. 196 (2009) 102-105, [arXiv: 0905.1198$].$

[8] T. Pierog, I. Karpenko, J. Katzy, E. Yatsenko, and K. Werner, “EPOS LHC : test of collective hadronization with LHC data”, arXiv:1306.0121.

[9] A. Ferrari, P. R. Sala, A. Fasso, and J. Ranft, "FLUKA: A multi-particle transport code (Program version 2005)”, tech. rep., CERN, 2005. CERN-2005-010, INFN/TC 05/11, SLAC-R-773.

[10] W. A. et al., "The spectrum of high-energy cosmic rays measured with KASCADE-Grande”, Astropart.Phys. 36 (2012), no. 1183 - 194.

[11] W. A. et al., "KASCADE-Grande measurements of energy spectra for elemental groups of cosmic rays”, Astropart.Phys. 47 (2013) 54-66, [arXiv:1306.6283]. 